\title{
Two cases of carfilzomib-induced thrombotic microangiopathy successfully treated with Eculizumab in multiple myeloma
}

Michael Rassner ${ }^{1 \dagger}$, Rebecca Baur ${ }^{2 \dagger}$, Ralph Wäsch ${ }^{1}$, Mario Schiffer ${ }^{3}$, Johanna Schneider ${ }^{4}$, Andreas Mackensen $^{2 \dagger}$ and Monika Engelhardt ${ }^{1 *+}$

\begin{abstract}
Background: Treatment with proteasome inhibitors like carfilzomib in patients with multiple myeloma (MM) can induce thrombotic microangiopathy (TMA) characterized by neurological symptoms, acute kidney injury, hemolysis and thrombocytopenia. Successful treatment with the monoclonal antibody eculizumab was described for these patients, but reports of ideal management and definitive treatment protocols are lacking.

Case Presentation: The first case describes a 43-years-old IgG-kappa-MM patient that developed TMA during the first course of carfilzomib-lenalidomide-dexamethasone (KRd) consolidation after autologous stem cell transplantation (ASCT). In the second case, a 59-years-old IgG-kappa-MM patient showed late-onset TMA during the fourth and last cycle of elotuzumab-KRd consolidation within the DSMM XVII study of the German study group MM (DSMM; clinicalTrials.gov Identifier: NCT03948035). Concurrently, he suffered from influenza A/B infection. Both patients had a high TMA-index for a poor prognosis of TMA. Therapeutically, in both patients plasma exchange (TPE) was initiated as soon as TMA was diagnosed. In patient \#1, dialysis became necessary. For both patients, only when the complement inhibitor eculizumab was administered, kidney function and blood values impressively improved.

(Continued on next page)
\end{abstract}

\footnotetext{
* Correspondence: monika.engelhardt@uniklinik-freiburg.de

${ }^{\dagger}$ Michael Rassner, Rebecca Baur, Andreas Mackensen and Monika Engelhardt contributed equally to this work.

'Department of Medicine I (Hematology, Oncology and Stem Cell

Transplantation), Medical Center, Faculty of Medicine, University of Freiburg, Hugstetter Strasse 53, D-79106 Freiburg, Germany

Full list of author information is available at the end of the article
}

(c) The Author(s). 2021 Open Access This article is licensed under a Creative Commons Attribution 4.0 International License, which permits use, sharing, adaptation, distribution and reproduction in any medium or format, as long as you give appropriate credit to the original author(s) and the source, provide a link to the Creative Commons licence, and indicate if changes were made. The images or other third party material in this article are included in the article's Creative Commons licence, unless indicated otherwise in a credit line to the material. If material is not included in the article's Creative Commons licence and your intended use is not permitted by statutory regulation or exceeds the permitted use, you will need to obtain permission directly from the copyright holder. To view a copy of this licence, visit http://creativecommons.org/licenses/by/4.0/ The Creative Commons Public Domain Dedication waiver (http://creativecommons.org/publicdomain/zero/1.0/) applies to the data made available in this article, unless otherwise stated in a credit line to the data. 


\begin{abstract}
(Continued from previous page)
Conclusion: In this small case series, two patients with MM developed TMA due to carfilzomib treatment (CFZ-

TMA), the second patient as a late-onset form. Even though TMA could have been elicited by influenza in the second patient and occurred after ASCT in both patients, with cases of TMA post-transplantation in MM being described, a relation of TMA and carfilzomib treatment was most likely. In both patients, treatment with eculizumab over two months efficiently treated TMA without recurrence and with both patients remaining responsive months after TMA onset. Taken together, we describe two cases of TMA in MM patients on carfilzomib-combination treatment, showing similar courses of this severe adverse reaction, with good responses to two months of eculizumab treatment.
\end{abstract}

Keywords: Multiple myeloma (MM), Thombotic microangiopathy (TMA), Eculizumab, Carfilzomib, Case report

\section{Background}

Thrombotic microangiopathy (TMA) is a rare cause of anemia in multiple myeloma (MM) patients, but can occasionally be elicited by common anti-myeloma drugs, especially by proteasome-inhibitors (PI) like carfilzomib (CFZ) [1-3]. Reports about TMA in MM induced by CFZ and optimal therapeutic approaches are scarce. Recently, single case reports described successful treatment of TMA in MM with the terminal complement pathway inhibitor eculizumab [1, 2, 4-6]. Definitive treatment recommendations, however, are lacking. Below we describe two cases of CFZ-induced TMA, that were successfully treated by eculizumab and provide evidence about precise therapeutic work-flow and possible interventions.

\section{Case 1}

A 43-year old male patient was diagnosed with IgGkappa-MM in August 2018. At admission, we found extensive osteolysis and an acute kidney failure with a serum-creatinine of $4.2 \mathrm{mg} / \mathrm{dl}$ [0.67-1.17] (GFR $15 \mathrm{ml} /$ min; MDRD) without previous history of impaired kidney function. With highly elevated serum free kappa light chains (kappa-SFLC) $3645 \mathrm{mg} / \mathrm{l}$ [0.35-15.10] and $4.2 \mathrm{~g} / \mathrm{d}[<0.15]$ urinary protein excretion, in the absence of albuminuria, cast nephropathy was assumed as the most likely cause of acute kidney failure. Since there was no sign of further organ injury, amyloidosis seemed unlikely, as well MIDD (monoclonal immunoglobulin deposition disease), which is often accompanied by amyloidosis and presents with albuminuria. The type of renal injury was not further determined by kidney puncture. The M-protein was elevated with $37 \mathrm{~g} / \mathrm{l}$, IgG $52.3 \mathrm{~g} / \mathrm{l}$ [6.1-16.16], k/l-ratio 864 [0.46-4], hemoglobin (Hb) $9.5 \mathrm{~g} / \mathrm{dl}$ [13.5-17.5], $\beta 2$-microglobulin $8.7 \mathrm{mg} / \mathrm{dl}$ [0-2.34], serum calcium and LDH were normal. The bone marrow plasma cell (BMPC) infiltration was 15\%, FISH excluded high-risk cytogenetics. Following the ISS grading system, we diagnosed an IgG-kappa-MM stage III (Durie \& Salmon stage IIIB) and symptomatic myeloma with 3/4 CRAB criteria (anemia, renal impairment, bone lesions). The revised myeloma comorbidity index (R-MCI: $1 / 9=$ fit/low-risk) revealed a fit patient [7-9]. Due to the CRAB-criteria, specifically renal impairment, chemotherapy was initiated with one cycle of bortezomib, doxorubicin, dexamethasone (BAD) followed by two cycles of bortezomib, cyclophosphamide, dexamethasone (VCD) after improvement of the kidney function and resolved urinary proteinuria. Since M-protein reduction was insufficient $(21.4 \mathrm{~g} / \mathrm{l})$, the regimen was switched a second time to CFZ, lenalidomide and dexamethasone (KRd). After two cycles KRd, a partial remission (PR; M-protein $5.3 \mathrm{~g} / \mathrm{l}, \mathrm{IgG} 6.4 \mathrm{~g} / \mathrm{l}$ ) was achieved and high-dose melphalan $\left(200 \mathrm{mg} / \mathrm{m}^{2}\right)$ chemotherapy with autologous stem cell transplantation (ASCT) was performed. On day (d) 42 post ASCT IgG levels were increasing again $(21.1 \mathrm{~g} / \mathrm{l})$. Based on the distinct improvement of the myeloma markers after KRd and a missing additional response to ASCT, our MM-tumor board decided to apply three consolidation cycles of KRd. In May 2019, the 3rd KRd cycle (1st consolidation cycle after ASCT) was initiated. At d2 of CFZ, the patient developed fever, dyspnea and malaise. Platelets were 11Tsd/ $\mu \mathrm{l}, \mathrm{Hb} 10.8 \mathrm{~g} / \mathrm{dl}, \mathrm{LDH}$ 1836U/l, haptoglobin < $150 \mathrm{mg} / \mathrm{l}$, unconjugated bilirubin $2.7 \mathrm{mg} / \mathrm{dl}$, the creatinine rose from 0.72 to $2.07 \mathrm{mg} / \mathrm{dl}$ [0.67-1.17] (GFR > 60 to $35 \mathrm{ml} /$ min) (Fig. 1a) and urinary proteinuria increased from normal values to $52 \mathrm{~g} / \mathrm{l}[<0.135]$. In the peripheral blood smear, $11 \%$ schistocytes were detected and an altered complement profile with reduced $\mathrm{C} 3$ and $\mathrm{C} 4$ levels was found (C3 77.6md/dl, C4 $9.9 \mathrm{mg} / \mathrm{dl}$ ). TMA-Index (LDH/ platelets ratio) was elevated to 166. These findings (schistocytes $>10 \%$, elevated creatinine and TMAIndex) have been described as poor prognostic factors for transplant associated-TMA (TA-TMA) [10]. Of note, two days earlier, lab results had been normal (Fig. 1a). Chest CT scan showed bilateral infiltrates, matching pulmonary hemorrhage (Fig. 2). Due to the acute development of hemolysis, together with acute kidney injury, unsuspicious ATAMTS13 activity (61\%) [50-110], normal ADAMTS13 antigen $(0.48 \mathrm{IU} / \mathrm{ml})[0.35-1.2]$ and direct Coombs test being negative, we suspected 

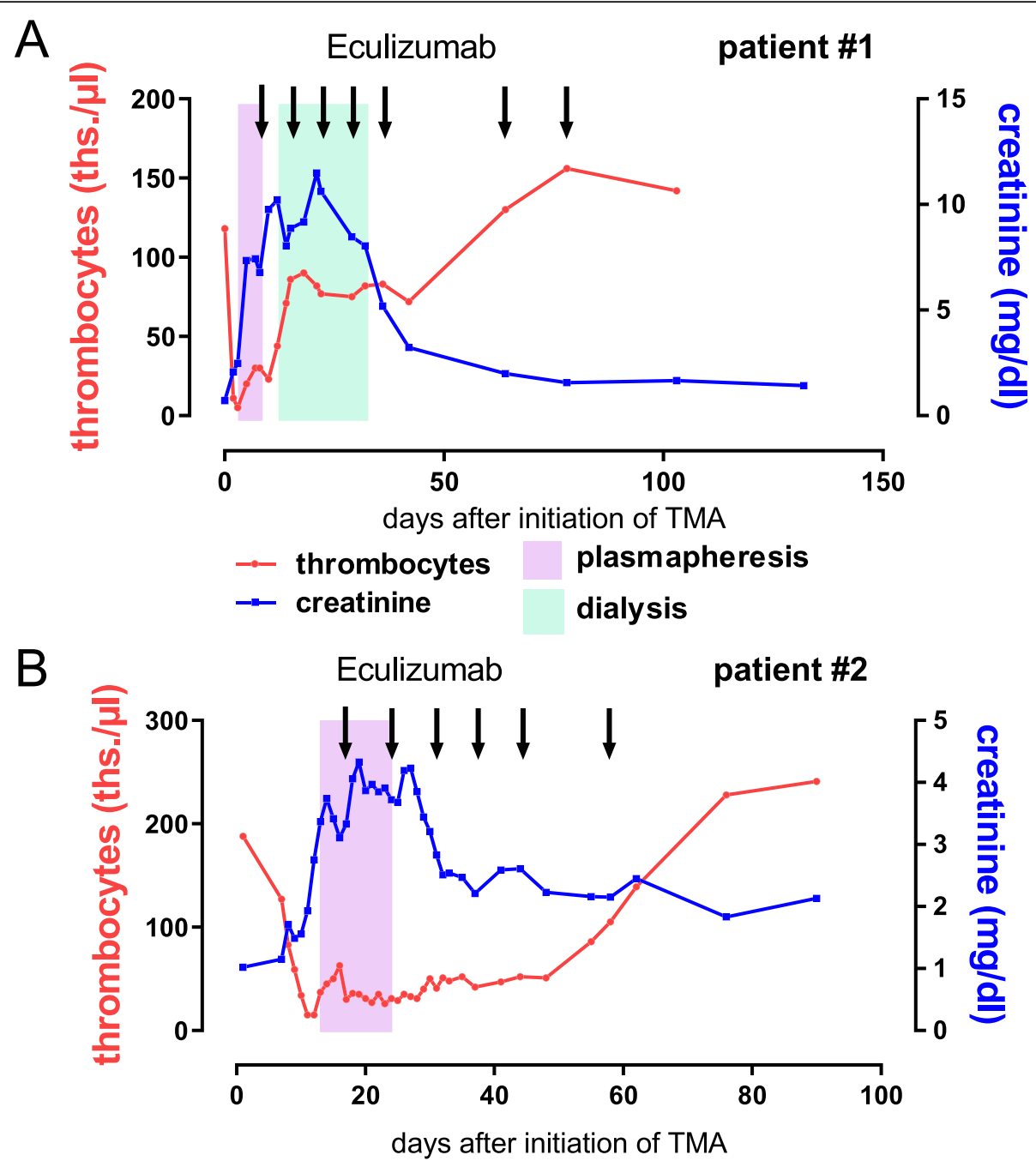

Fig. 1 Course of CFZ-TMA in the two patients. a Case \#1: This patient developed acute kidney injury, fever and pulmonary hemorrhage during the first consolidation with carfilzomib, lenalidomide and dexamethasone (KRd) after ASCT. Laboratory findings (thrombocytopenia) and schistocytosis of $11 \%$ were consistent with thrombotic microangiopathy (TMA). Therapeutic plasma exchange (TPE) was immediately initiated, but shortly after dialysis was necessary. As blood values did not improve, we finally administered eculizumab for a total of seven doses and saw a rapid response in $\mathrm{LDH}$, platelet number and kidney function with our patient being still in PR. $\mathbf{b}$ Case \#2: This patient, who is treated in the DSMM XVII study (Arm A: Elotuzumab-KRd) developed head ache, malignant hypertension and laboratory distortions (thrombocytopenia, increase of LDH and acute kidney injury) consistent with TMA. After a short period of TPE, eculizumab was infused (total of six infusions). Blood values improved but kidney function remains decreased

medication-induced TMA. Therapeutic plasma exchange (TPE) was initiated by replacement with fresh frozen plasma (FFP) on a daily regimen and on account of declining kidney function with development of acute kidney injury stage 3 according to the acute Kidney Injury Network (AKIN), dialysis was necessary one day later. After six days of TPE neither LDH nor platelets improved. Assuming CFZ-induced TMA, we administered eculizumab $(900 \mathrm{mg}$ ) along with meningococcal vaccination and initiation of antibiotic prophylaxis. Five days later, LDH declined to $818 \mathrm{U} / \mathrm{l}$ and platelet counts improved to $58 \mathrm{Tsd} / \mu \mathrm{l}$ without transfusions. We continued eculizumab (900 mg) weekly. After the fourth application, dialysis could be discontinued. Platelet counts normalized within two months after treatment initiation of eculizumab and there were no further signs of TMA, thus eculizumab was terminated (Fig. 1a). One year after TMA diagnosis and KRd consolidation, our patient remains in PR of his MM and CR of the CFZinduced TMA.

\section{Case 2}

The second 59-year-old male patient was diagnosed with IgG-kappa-MM in 11/2018. CT scan revealed osteolysis of the sacrum, pelvis and vertebrae. Calcium and creatinine were normal and the $\mathrm{Hb}$ was slightly decreased 

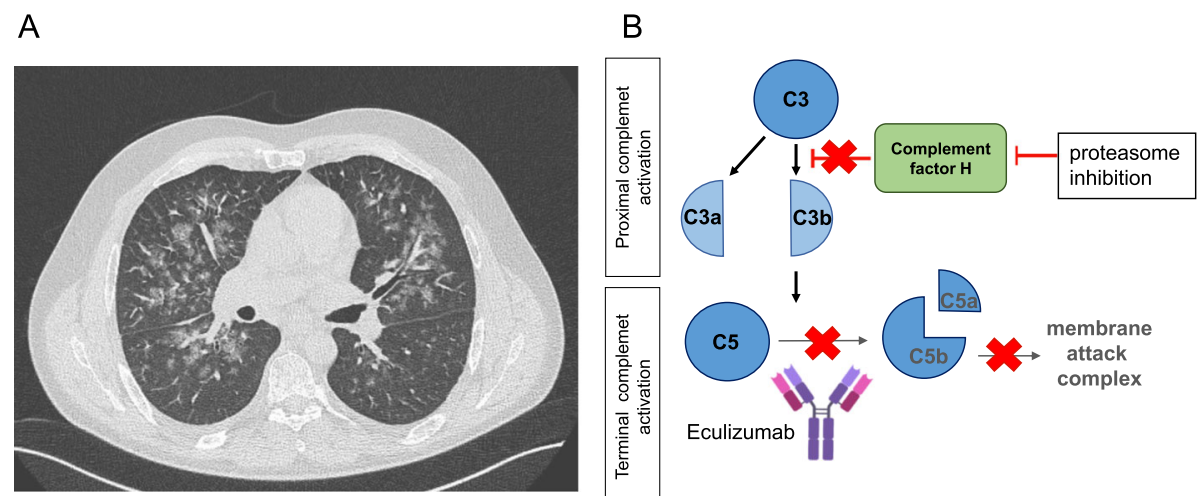

Fig. 2 a Chest-CT in patient case 1, d1 of CFZ-TMA onset: Bipulmonary extensive ground-glass opacities in all lung fields, mostly omitting the periphery and predominantly occurring in the right lung. $\mathbf{b}$ Mechanism of CFZ-TMA: Within the proximal complement activation C3 is cleaved by the C3 convertase to anaphylactic 3a and C3b. C3b then cleaves $C 5$ to C5a and C5b, the latter together with C6-C9 forming the membrane attack complex (MAC) within the terminal pathway. Activity of the $\mathrm{C} 3$ convertase is regulated by complement factor $\mathrm{H}$ and regulated proteins Proteasome inhibition blocks CFH [1 1]. The monoclonal antibody eculizumab counteracts the resulting overactivation of C3b by binding C5

(13.3 g/dl). IgG was $38.4 \mathrm{~g} / \mathrm{l}, \mathrm{k}-\mathrm{SFLC} 182 \mathrm{mg} / \mathrm{l}, \mathrm{k} / \mathrm{l}$-ratio 60.5. BM biopsy revealed 40\% PC infiltration. Cytogenetics were favorable (trisomy 5p15, 9q22, 11q13 und 15q22). The ISS and R-ISS were both I, Durie \& Salmon stage was IIIA and symptomatic MM was diagnosed with $1 / 4$ CRAB symptoms. The patient was classified as fit for ASCT (R-MCI-score: 2/9 = fit) [7-9]. We included the patient into the DSMM XVII study of the German study group MM (DSMM; clinicalTrials.gov Identifier: NCT03948035). Induction with elotuzumab, CFZ, lenalidomide and dexamethasone (E-KRd, 6 cycles) and ASCT with melphalan $200 \mathrm{mg} / \mathrm{m}^{2}$ were performed and due to repeated respiratory infections (boca- and corona $(\mathrm{CoV}$ NL 63) virus) dose reductions of elotuzumab and lenalidomide were executed. Before ASCT, kappa-SFLC decreased to $12.8 \mathrm{mg} / \mathrm{l}$ (vgPR).

In 01/20, within the last (4th) cycle of E-KRd consolidation, the patient developed fever and acute kidney failure with creatinine $1.7 \mathrm{mg} / \mathrm{dl}$ (GFR $40 \mathrm{ml} / \mathrm{min}$, MRDRD; baseline-creatinine: $1 \mathrm{mg} / \mathrm{dl}$ ). Prerenal genesis was assumed (fractional sodium excretion $<1 \%$ ) and fluids were infused. However, creatinine elevation did not resolve finally reaching acute kidney injury stage 3 according to AKIN and proteinuria reached $6.2 \mathrm{~g} / \mathrm{d}$. The patient developed headache and high blood pressure (BP 220/105 mmHg). Platelets and Hb gradually decreased (Fig. 1b), haptoglobin was $<5 \mathrm{mg} / \mathrm{dl}$. In the peripheral blood smear, $44 \%$ schistocytes were visible; ADAMTS13 activity and antigen were normal $(58 \%$ and $0.40 \mathrm{IU} / \mathrm{ml}$, respectively) and Coombs test negative. Blood culture additionally showed Staphylococcus epidermidis due to port infection and throat swab detected influenza A (H3N2) and B.

Since TMA was diagnosed (LDH/platelets ratio 88.3), high-dose glucocorticoids and TPE were applied (before completion of diagnostics). Without substantial improvement, five days after first plasmapheresis our patient received eculizumab (Fig. 1b), starting at $900 \mathrm{mg}$. A total of 11 sessions of plasma exchange at a daily basis were performed with FFP as replacement (Fig. 1b). Parameters of hemolysis rapidly improved, so that glucocorticoids could be expeditiously reduced and eculizumab was terminated after four cycles with $900 \mathrm{mg} /$ weekly and two doses with $1200 \mathrm{mg} /$ every two weeks (Fig. 1b).

In $05 / 19$, the patient showed VGPR of his MM, the BMPC infiltration even CR (polyclonal PCs of 5\%) and gradual renal function improvement (eGFR improved from 10 to $32 \mathrm{ml} / \mathrm{min}$ ). Maintenance therapy with reduced doses of elotuzumab-lenalidomide (E-R: lenalidomide with $5 \mathrm{mg}$ d1-28) was successfully initiated and TMA has remained in CR.

\section{Discussion and conclusion}

Hemolysis is a rather rare cause for anemia in MM [12]. When anemia is accompanied with thrombocytopenia, acute kidney injury, high blood pressure, neurological or unexplained extrarenal symptoms TMA should be considered. TMA includes a group of etiologically diverse diseases: Primary TMA, which include atypical HUS (aHUS) or thrombotic thrombocytopenic purpura (TTP) and secondary TMA and infection-associated TMA, involving Shiga-toxin-producing Escherichia coliassociated hemolytic uremic syndrome (STEC-HUS) $[6$, 13, 14]. TTP, in contrast to other TMA forms, is defined by decreased activity of the vWF-cleaving enzyme ADAMTS13 [6]. Unlike "typical" HUS, which is triggered by infections with STEC, complement mediated TMA is driven by uncontrolled complement activation and affected patients frequently harbor mutations in 
complement regulatory proteins (Fig. 2b) [15]. Secondary TMA is elicited by medication, malignancy, pregnancy, malignant hypertension, transplantations, infections or associated to autoimmune diseases $[6,10$, 12-14]. As reviewed recently [6], TMA in MM can be elicited by ASCT- or allogeneic-SCT, MM progression, or anti-myeloma therapy. Drug-induced TMA (DITMA) may occur in MM due to PIs bortezomib or CFZ [3, 16, 17]. CFZ-TMA can appear early after onset of the drug, but also as late as two years after first administration [1, 2]. CFZ-TMA is probably driven by complement activation of the alternative pathway in terms of complementmediated TMA $[2-4,18]$. Blasco et al. described three cases in which plasma samples from patients with CFZTMA, led to depositions of complex membrane attack complex (C5b-9) of on endothelial cells, suggesting a complement overactivation [4]. Supporting this assumption, proteasome inhibition has been found to block Factor $\mathrm{H}$, consequently leading to complement activation (Fig. 2b) [11]. Bortezomib-induced cases of TMA are attributed to decreased activity of ADAMTS13 [19, 20]. However, the majority of bortezomib-induced cases of TMA/TTP had only slightly decreased ADAMTS13 activity and PI-induced TMA in MM might be generally based on pathological overactivation of the alternative complement pathway rather than TTP [6]. In patient \#2, TMA was either induced by CFZ or influenza A or B $[15,21]$. Also a combination of both preexistent vascular endothelial injury due to CFZ and transient complement activation during influenza infection was eagerly discussed within our group. In this respect, eculizumab could be effective in patients treated with concurrent PI treatment, infection and TMA.

In patient \#2, in contrast to patient \#1, C3 and C4 levels were normal; $\mathrm{CH} 50$ and $\mathrm{C} 5 \mathrm{~b}-9$, the terminal pathway of the complement pathway were, however, not assessed. The fast response to eculizumab and the lacking effect of TPE seemed to argue for pathological complement activation, but it cannot be ruled out that the classical or the alternative complement pathway was involved. Importantly, both patients developed TMA several weeks after ASCT. Since TA-TMA was described to occur in a complement-dependent manner, like complement mediated TMA [10], a combination of TMAtriggers is likewise conceivable.

Taken together, several factors might have induced TMA in our patients: ASCT, genetic predispositions, viral infections and the underlying CFZ treatment. Thus, definite causality could ot be entirely proven. The major criticism of our study may be missing additional in vitro studies of complement activation and the unavailability of renal biopsies for further diagnostics. Kidney biopsies had been discussed interdisciplinary in our weekly MM tumor board, however, in the acute clinical setting, the potential biopsy risk, especially bleeding, was judged to outweigh potential benefits and on top, both patients had declined kidney biopsies.

In respect to therapy for TMA in MM, definitive clinical practical guidelines do not exist [6]. For DITMA, the causative drug must be discontinued promptly, highdose glucocorticoids are given and plasmapheresis is performed [2, 6]. In CFZ-TMA, successful treatment with eculizumab, a terminal complement pathway inhibitor, was described in six cases to our knowledge [1, $2,4,5,22]$. Nevertheless, reports of TMA in MM remain scarce, and the definitive treatment regimen as well as definitive duration of treatment are unclear [6]: Portuguese and Lipe (2018), Moliz et al. (2019) and Blasco et al. (2020) administered eculizumab for up to three weekly doses [2, 4, 22], whereas Bhutani et al. (2020) for four months [1]. In our two cases, only after eculizumab initiation, hemolysis and creatinine levels improved impressively (Fig. 1A,B), plus the application period of two months of eculizumab was efficient without relapsing TMA, thus appears possible to be shorter than four months. On the other hand a shorter treatment period, as reported by Portuguese and Lipe, might be insufficient as one of their eculizumab-treated patients stayed on hemodialysis with this regime [22].

TPE is generally recommended for TMA. Plasma exchange, however, is not without side effects, and can remove therapeutic antibodies from the circulation, thus its concurrent use is not recommended [6, 10]. Interestingly, in our cases the success of eculizumab was not fully dependent on the termination of TPE, since in patient \#2 TPE was continued during the first two eculizumab doses. In this respect, concerns, that TPE would remove eculizumab from the blood or restore C5-levels available for activation, seem unfounded. On the other hand, a significant improvement of creatinine-levels and platelets was only achieved after termination of TPE in patient \#2 (Fig. 1B). Thus, irrespective of the cause of TMA in MM patients under PI treatment or shortly after ASCT, eculizumab rather than TPE could be considered as standard first-line therapy [2,6] (Fig. 3). Increased susceptibility to meningococcal infection during eculizumab treatment has to be considered and vaccination should be implemented [23]. As soon as ADAMTS13 activity is proven to be within the normal range $(50-150 \%)$ and TTP is excluded, further treatment decisions can be drawn [6]. Additionally, in our experience short-term use of eculizumab was sufficient for effective TMA treatment. Furthermore, eculizumab treatment did not worsen MM parameters in neither of our patients.

Taken together, CFZ-induced TMA is a serious event and should be assumed in treated MM patients with 


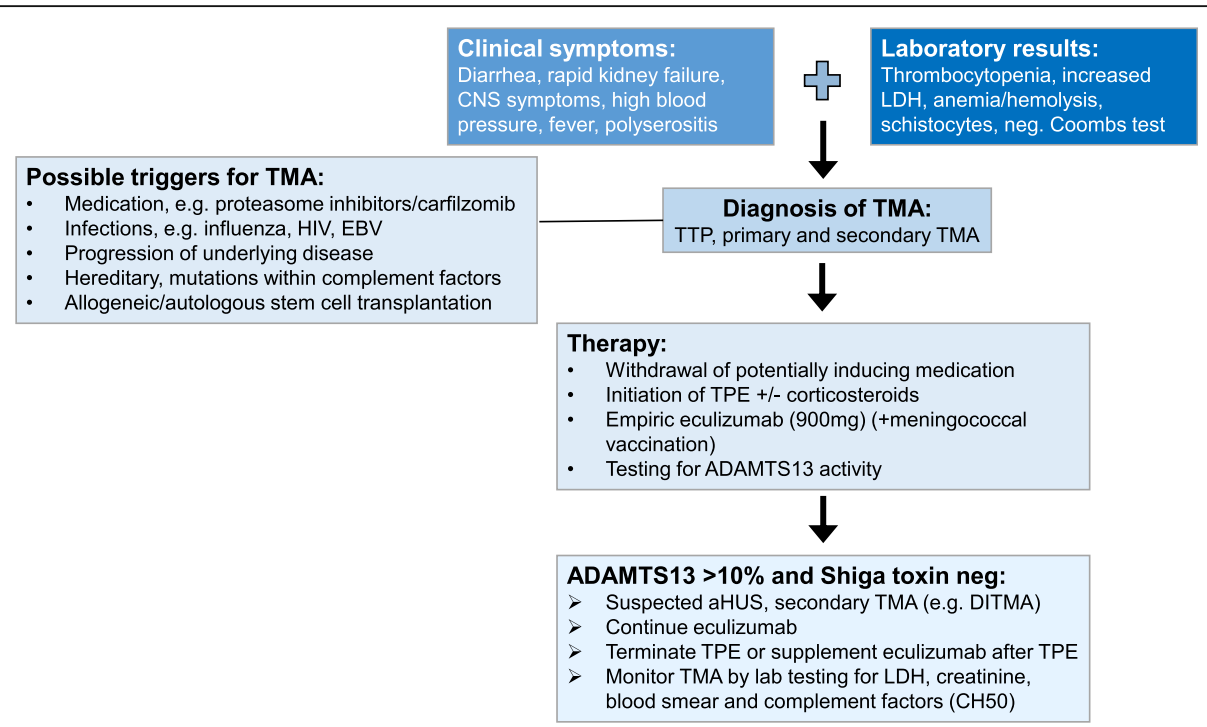

Fig. 3 Guideline for diagnostic and therapeutic approach for TMA in MM: Whenever clinical and laboratory findings are consistent with TMA, one should rapidly terminate possible causative drugs, initiate therapeutic plasma exchange (TPE) and apply a first dose of eculizumab. As soon as ADAMTS13 activity is determined further therapeutic decisions can be drawn

rapid acute kidney failure and hemolysis. Fast treatment initiation with eculizumab can reverse pathological complement system activation and prevent organ damage. As shown in our patients, early initiated, short-term treatment with eculizumab can sufficiently terminate CFZ-TMA and counteract TMA-poor prognostic factors.

\section{Abbreviations}

aHUS: Atypical hemolytic uremic syndrome; AKIN: Acute kidney injury network; ASCT: Autologous stem cell transplantation; BM: Bone marrow; CFZTMA: Carfilzomib-induced thrombotic microangiopathy; CR: Complete response; DITMA: Drug-induced thrombotic microangiopathy; FFP: Fresh frozen plasma; TMA: Thrombotic microangiopathy; TPE: Therapeutic plasma exchange; TTP: Thrombotic thrombocytopenic purpura; MM: Multiple myeloma; PC: Plasma cell; PI: Proteasome inhibitor; STEC: Shiga-toxinproducing E.coli; vgPR: Very good partial response

\section{Acknowledgements}

We would like to thank both patients for consent of publication of their educational cases within this work.

\section{Authors' contributions}

$M R$ and RB treated the patients, drafted and wrote the manuscript; JS, MS and RW treated the patient and revised the manuscript; AM and ME treated the patient, initiated and wrote the manuscript. All authors read and approved the final manuscript.

\section{Funding}

There was no funding received to support this research. Open Access funding enabled and organized by Projekt DEAL.

\section{Availability of data and materials}

Not applicable.

Ethics approval and consent to participate Not applicable.

\section{Consent for publication}

We have obtained written consent for publication from both patients presented in this case report.

\section{Competing interests}

The authors declare that they have no competing interests.

\section{Author details}

'Department of Medicine I (Hematology, Oncology and Stem Cell Transplantation), Medical Center, Faculty of Medicine, University of Freiburg, Hugstetter Strasse 53, D-79106 Freiburg, Germany. ${ }^{2}$ Department of Internal Medicine 5 - Hematology/Oncology, University Hospital of Erlangen, Erlangen, Germany. ${ }^{3}$ Department of Internal Medicine 4 - Nephrology, University Hospital of Erlangen, Erlangen, Germany. ${ }^{4}$ Department of Medicine IV, Medical Center, Faculty of Medicine, University of Freiburg, Freiburg, Germany.

Received: 16 July 2020 Accepted: 25 December 2020

Published online: 18 January 2021

\section{References}

1. Bhutani D, Assal A, Mapara MY, Prinzing S, Lentzsch S. Case Report: Carfilzomib-induced Thrombotic Microangiopathy With Complement Activation Treated Successfully With Eculizumab. Clin Lymphoma Myeloma Leuk. 2020;20:e155-7.

2. Moliz C, Gutiérrez E, Cavero T, Redondo B, Praga M. Eculizumab as a treatment for atypical hemolytic syndrome secondary to carfilzomib. Nefrologia. 2019:39:86-8.

3. Mehta N, Saxena A, Niesvizky R. Bortezomib-induced thrombotic thrombocytopaenic purpura. BMJ Case Rep. 2012;2012

4. Blasco M, Martínez-Roca A, Rodríguez-Lobato LG, Garcia-Herrera A, Rosiñol $L$, Castro P, et al. Complement as the enabler of carfilzomib-induced thrombotic microangiopathy. Br J Haematol. 2020.

5. Gosain R, Gill A, Fuqua J, Volz LH, Kessans Knable MR, Bycroft R, et al. Gemcitabine and carfilzomib induced thrombotic microangiopathy: eculizumab as a life-saving treatment. Clinical Case Reports. 2017;5:1926-30.

6. Portuguese AJ, Gleber C, Passero FC, Lipe B. A review of thrombotic microangiopathies in multiple myeloma. Leuk Res. 2019;85:106195.

7. Engelhardt M, Dold SM, Ihorst G, Zober A, Moller M, Reinhardt $\mathrm{H}$, et al. Geriatric assessment in multiple myeloma patients: validation of the International Myeloma Working Group (IMWG) score and comparison with other common comorbidity scores. Haematologica. 2016;101:1110-9. 
8. Engelhardt M, Domm A-S, Dold SM, lhorst G, Reinhardt H, Zober A, et al. A concise revised Myeloma Comorbidity Index as a valid prognostic instrument in a large cohort of 801 multiple myeloma patients. Haematologica. 2017;102:910-21.

9. Engelhardt M, Ihorst G, Duque-Afonso J, Wedding U, Spät-Schwalbe E, Goede V, et al. Structured assessment of frailty in multiple myeloma as a paradigm of individualized treatment algorithms in cancer patients at advanced age. Haematologica. 2020;105:1183-8.

10. Khosla J, Yeh AC, Spitzer TR, Dey BR. Hematopoietic stem cell transplantassociated thrombotic microangiopathy: current paradigm and novel therapies. Bone Marrow Transplant. 2018:53:129-37.

11. Liu Z, Qin T, Zhou J, Taylor A, Sparrow JR, Shang F. Impairment of the Ubiquitin-Proteasome Pathway in RPE Alters the Expression of Inflammation Related Genes. Adv Exp Med Biol. 2014;801:237-50.

12. Rassner M, Jung J, Schneider J, Wäsch R, Engelhardt M. Dapsone-induced hemolytic anemia in multiple myeloma - a case report of various differential diagnoses. Clinical Lymphoma Myeloma and Leukemia. 2020; S2152265020303190

13. Aigner C, Schmidt A, Gaggl M, Sunder-Plassmann G. An updated classification of thrombotic microangiopathies and treatment of complement gene variant-mediated thrombotic microangiopathy. Clinical Kidney Journal. 2019;12:333-7.

14. Brocklebank V, Wood KM, Kavanagh D. Thrombotic Microangiopathy and the Kidney. Clinical journal of the American Society of Nephrology: CJASN. 2018;13:300-17.

15. van Hoeve K, Vandermeulen C, Van Ranst M, Levtchenko E, van den Heuvel L, Mekahli D. Occurrence of atypical HUS associated with influenza B. Eur J Pediatr. 2017;176:449-54

16. Haddadin M, Al-Sadawi M, Madanat S, Tam E, Taiwo E, Luhrs C, et al. Late Presentation of Carfilzomib Associated Thrombotic Microangiopathy. Am J Med Case Rep. 2019;7:240-3.

17. Yui JC, Van Keer J, Weiss BM, Waxman AJ, Palmer MB, D'Agati VD, et al. Proteasome inhibitor associated thrombotic microangiopathy. Am J Hematol. 2016;91:E348-52.

18. Caprioli J, Noris M, Brioschi S, Pianetti G, Castelletti F, Bettinaglio P, et al. Genetics of HUS: the impact of MCP, CFH, and IF mutations on clinical presentation, response to treatment, and outcome. Blood. 2006;108:1267-79.

19. Chan K-L, Filshie R, Nandurkar H, Quach H. Thrombotic microangiopathy complicating bortezomib-based therapy for multiple myeloma. Leuk Lymphoma. 2015;56:2185-6.

20. Pettitt $A R$, Clark RE. Thrombotic microangiopathy following bone marrow transplantation. Bone Marrow Transplant. 1994;14:495-504.

21. Bitzan M, Zieg J. Influenza-associated thrombotic microangiopathies. Pediatr Nephrol. 2018;33:2009-25.

22. Portuguese AJ, Lipe B. Carfilzomib-induced aHUS responds to early eculizumab and may be associated with heterozygous CFHR3-CFHR1 deletion. Blood Adv. 2018;2:3443-6.

23. Crew PE, McNamara L, Waldron PE, McCulley L, Jones SC, Bersoff-Matcha SJ. Unusual Neisseria species as a cause of infection in patients taking eculizumab. J Infect. 2019:78:113-8.

\section{Publisher's Note}

Springer Nature remains neutral with regard to jurisdictional claims in published maps and institutional affiliations.

Ready to submit your research? Choose BMC and benefit from:
- fast, convenient online submission
- thorough peer review by experienced researchers in your field
- rapid publication on acceptance
- support for research data, including large and complex data types
- gold Open Access which fosters wider collaboration and increased citations
- maximum visibility for your research: over 100M website views per year
At BMC, research is always in progress.
Learn more biomedcentral.com/submissions

